\title{
Automated Generalization of Translation Examples
}

\author{
Ralf D. Brown \\ Carmegie Mellon University, Language T'echnologies Institute \\ Pittsburgh, l'A 15213-3890 \\ ralf'+(i)cs.cmu.cdu
}

\begin{abstract}
Previous work has shown that adding generalization of the examples in the corpus of an example-based machine translation ('BSM'T) system can reduce the reguired amount of pretranslated example text by as much as an order of magnitude for Spanish-jinglish and lirenchInglish l'Bl3M'l. Using word clustering to antomatically generalize the example corpus can provide the majority of this improvement for lirench-Finglish with no mannal intervention; the prior work recuired a large bilingual dictionary lagged with parts of speed and the manual creation of grammar rules. By secoling the clustering with a small amount of manuallycreated information, even betier performance can be achicved. This paper describes a method whereby bilingual word clustering can be performed using standased monolingual document, clustering technicuues, and its eflectiveness at reducing the size of the example corpus required.
\end{abstract}

\section{Introduction}

lixample-Based Machine 'Translation (EBMT') relies on a collection of textual units (usually' sentences) and their translations. New text to be translated is matched against the sourcelanguage half of the collection, and the corresponding translations from the target-languago lialf are used to generate a translation of the new text.

Experience with several language pairs has shown that producing an lisMM'T' system which provides reasonable translation coverage of unrestricted texts using simple textual matching requires on the order of two million words of pre-translated texts (one million words in cach language); if either language is highly inflecting, polysynthetic, or (worse yet) agglutinative, even more text will be required. It may be difficult, time-consuming, and expensive to obtain that much parallel text, particularly for lesser-used language pairs. 'Thus, if one wishes to develop a new translator rapidly and at, low cost, techniques are needed which permit the EBMM'T sys- tem to perform just as well using substantially lesis example text.

Both the Gaijin CBMM'l system by Veale and Way (1997) and the author's EBM'I' system (1999) convert the examples in the corpus into templates against which the new texts can be matched. Gaijin variablizes the well-formed segment mappings between source and target sentences that it is able to find, using a closed set of marker's to segment the input into phrases. T'lo author's system performs its generalization using equivalence classes (both syntactic and somantic) and a production-rule grammar. liast, any occurrences of terms contained in an equivalence class are replaced by a token giving the name of the equivalence class, and then the grammar rules are used to replace patterns of words and tokens by more general tokens (such as $\left\langle\mathrm{NI}^{3}\right\rangle$ for noun phrases). (Brown, 1999) showed that one can reduce the corpus size by as much as an order of magnitude in this way.

(iven that explicit, manually-generated equivalence classes reduce the need for example text, an obvious extension would be to attempt to gonerate these classes automatically from the corpus of pro-translated examples. T'his paper describes one approach to automated extraction of equivalence classes, using clustering tecliniques.

The remainder of this paper describes how 1o perform bilingual word clustering using standard monolingual document clustering technicques by converting the problem space; the various clustering algorithms which were investigated; and the effectiveness of generalization using the derived clusters at reducing the required amount of example text.

\section{Converting the Problem}

T'he task of clustering words according to their occurrence patterns can be restated as a standard document-clustering task by converting the problem space. lior each unique word to be clustered, create a pseudo-document, containing the words of the contexts in which that word ap- 
pears, and use the word itself as the document identifier. After the pseudo-documents are clustered, retrieving the identifier for eacl document in a particular cluster produces the list of words occurring in sufficiently similar contexts to be considered equivalent for the purposes of generalizing an EBMT system.

By itself, this approach only produces a monolingual clustering, but we require a bilingual clustering for proper generalization since different senses of a word will appear in differing contexts. 'The method of Barrachina and Vilar' (1999) provides the means for injecting bilingual information into the clustering process.

Using a bilingual dictionary -- which may bo created from the corpus using statistical methods, such as those of Peter Brown el al (1990) or the author's own previous work (Brown, 1997) - and the parallel text, create a rough mapping between the words in the source-language half of cach translation example in the corpus and the target-language half of that example. Whenever there is exactly one possible translation candidate listed for a word by the mapping, generate a bilingual word pair consisting of the word and its translation. This word pair will be treated as an indivisible token in further processing, adding bilingual information to the clustering process. Forming pairs in this manner causes each distinct translation of a word to be treated as a separate sense; although translation pairs do not cxactly correspond to word senses, pairs can be formed without any additional knowledge sources and are what the EBM I' system requires for its equivalence classes.

For every unique word pair found in the previous step, we accumulate counts for each word in the surrounding context of its occurrences. The context of an occurrence is defined to be the $N$ words immediately prior to and the $N$ words immediately following the occurrence; $N$ currently is set to 3 . Because word order is important, counts are accumulated separately for each position within the context, i.c. for $N=3$, a particular context word may contribute to any of six different counts, depending on its location relative to the occurrence. Further, as the distance from the occurrence increases, the surrounding words become less likely to be a true part of the word-pair's context, so the counts are weighted to give the greatest importance to the words immediately adjacent to the word pair being examined. Currently, a simple lincar decay from 1.0 to $\frac{1}{N}$ is used, but other decay functions such as the reciprocal of the distance are also possible. 'The resulting weighted set of word counts forms the above-mentioned pseudodocument which is converted into a term vector for cosine similarity computations (a standard measure in information retrieval, defined as the dot product of two term vectors normalized to unit length).

If the clustering is seeded with a set of initial equivalence classes (which will be discussed below), then the equivalences will be used to generalize the contexts as they are added to the overall counts for the word pair. Any words in the context for which a unique correspondence can be found (and for which the word and its corresponding translation are one of the pairs in an equivalence class) will be counted as if the name of the equivalence class had been present in the text rather than the original word. For example, if days of the week are an equivalence class, then "did he come on Friday" and "did he loave on Monday" will yicld identical context vectors for "come" and "leave", making it easier for those two terms to cluster together.

'lo illustrate the conversion process, consider the French word "cing" in two cxamples where it translates into English as "five" (thus forming" the word pair "cinq-five"):

$\left\langle N U I_{\rangle}\right\rangle\left\langle N U I_{i}\right\rangle$ le cinq jours depuis la

$\langle N U L\rangle\langle N U L\rangle$ The five days since the

elles commenceront en cinq jours . $\langle N U L\rangle$ they will begin in five days. $\left\langle N U I_{s}\right\rangle$ where $<$ NUI $>$ is used as a placcholder. when the word pair is too near the beginning or end of the sentence for the full context to be present. Note that the word order on the target-language side is not considered when building the term vector, so it need not be the same as on the source-language side; the examples were chosen with the same word order merely for clarity.

The resulting term vector for "cinc_five" is as follows, where the numbers in parentheses indicate the context word's position relative to the word pair under consideration:

\begin{tabular}{lcr} 
Word & Occur & Weight \\
\hline$\langle$ NUL $>(-3)$ & 1 & 0.333 \\
clles $(-3)$ & 1 & 0.333 \\
$\langle$ NUI $\rangle(-2)$ & 1 & 0.667 \\
commenceront $(-2)$ & 1 & 0.667 \\
Le $(-1)$ & 1 & 1.000 \\
cn $(-1)$ & 1 & 1.000 \\
jours $(1)$ & 2 & 2.000 \\
depuis $(2)$ & 1 & 0.667 \\
(2) & 1 & 0.667 \\
la $(3)$ & 1 & 0.333 \\
$\langle$ NUL $>(3)$ & 1 & 0.333
\end{tabular}

Term vectors such as the above are then clustered to determine equivalent usages among words. 


\section{Clustering Approaches}

A total of six clustering algorithms have been tested; three vartants of group-average clustering and three of agglomerative clustering. Incremental group-average clustering was implemented first, to provide a proof of concept, before the computationally more expensive agglomerative (bottom-up) clustering was impleinented.

'The incremental group-average algorithms all examine each word pair in turn, computing a similarity moasure to every existing cluster. If the best similarity measure is above a predetermined threshold, the new word pair is placed in the corresponding cluster; otherwise, an new chuster is created. 'The throe variants differ only in the sinilarity measure employed:

1. cosine similarity between the pseudo-docunent and the centroid of the existing cluster (standard group-average clustering)

2. average of the cosine similarities between the preudo-document and all members of the existing cluster (average-link clustering)

3. square root of the average of the squared cosine similarities between the pseudodocument, and all members of the existing chuster (root-moan-scpuare modification of average-link clustering)

These three variations give increasingly more weight to the nearer members of the existing cluster.

'The bottom-tup agglomerative algorithms all function by creating a cluster for ach pseudodocument, then repeatedly morging the two clusters with the highest similarity score until no two clusters have a similarity score exceding a predetermined theshold. 'The three variants again differ only in the similarity measure employed:

1. cosine similarity between cluster controids (standard agglomerative clustering)

2. average of cosine similarity between mombers of the two clusters (average-link)

3. maximal cosine similarity between any pair of members of the two clusters (single-link)

fior each of the variations above, the predetermined threshokt is a function of word frequency. 'T'wo words which each appear only once in the entire training text and have a high similarity score are more likely to have appeared in similar contexts by coincidence than two words which each appear in the training text fifty times.

\begin{tabular}{|c|c|}
\hline Trequency & Threshold \\
\hline 1 & 1.00 \\
2 & 0.85 \\
3 & 0.80 \\
1 & 0.75 \\
5 & 0.70 \\
6 & 0.65 \\
7 & 0.60 \\
$8-9$ & 0.55 \\
$10-11$ & 0.50 \\
$12-15$ & 0.45 \\
$\geq 16$ & 0.40 \\
\hline
\end{tabular}

ligure 1: Clustering 'I'hreshold liunction

For example, when using three words on either side as context, and a lincar decay in term weights, two singleton words achicve a similarity score of 0.321 (1.000) is the maximum possible) if just one of the immediately adjacent words is the same for both, even if none of the other five context words are the same. As the number of occurrences increases, the contribution to the similarity score of individual words decreases, making it less likely to cncounter a high score by chance. Hence, we wish to set a stricter threshold for clustering low-frecuency words than higher-frecuency words.

the threshold function is expressed in terms of the frequency of occurrence in the training texts. For single, nnclustered word pairs, the frepuency is simply the number of times the word pair was enconntered. When performing group-average clustering, the frequency assigned to a cluster is the sum of the frequencies of all the members; for agglomerative clustering, the frequency is the sum when using controids and the maximum frequency among the members when nsing the average or nearest-neightor similarity. The wahe of the threshold for a given pair of clusters is the value of the threshold function at the lower word frequency. Figure 1 shows the threshold function used in the experinents whose results are reported here; clustering is only allowed if the similatity measure is above the indicated threshold value.

On its own, clustering is quite successful for generalizing EBMT examples, but the fullyautomated production of clusters is not compatible with adding a production-rule grammar as described in (Brown, 1999). 'Therefore, the clustering process may be seeded with a set of manually-generated clusters.

When seed clusters are available, the clustering process is modified in two ways. First, the group-average approaches add an initial cluster for each seed cluster and the agglomerative ap- 
proaches add an initial cluster for each word pair; these initial clusters are tagged with the name of the seed cluster. Sccond, whenever a tagged cluster: is merged with an untagged one or another cluster with the same tag, the combination inherits the tag; further, merging two clusters with different tags is disallowed. As a result, the initial seed clusters are expanded by adding additional word pairs while preventing any of the seed clusters from themselves merging with cach other.

One special case is handled separately, namely numeric strings. If both the sourcelanguage and target-language words of a word pair are numeric strings, the word pair is treated as if it had been specified in the seed class $<$ number $>$. Word pairs not containing a digit in eithor word can optionally be prevented from being added to the <number> cluster unless explicitly secded in that cluster. The former feature ensures that numbers will appear in a single cluster, rather than in multiple clusters. The latter avoids the inclusion of the many nonnumeric word pairs (primarily adjectives) which would otherwise tend to cluster with numbers, because both they and numbers are used as modifiers.

Once clustering is completed, any clusters which have inherited the same lag (which is possible when using agglomerative clustering) are merged. 'Those clusters which contain more than one pscudo-document are output, together with any inherited label, and can be used as a set of equivalence classes for $\mathrm{HBM}^{\mathrm{T}} \mathrm{T}$.

Agglomerative clustering using the maximal cosine similarity (single-link) produced the subjectively best clusters, and was used for the experiments described here.

\section{Experiment}

The method described in the previous two sections was tested on French-] English EBMT. The training corpus was a subset of the IBM Hansard corpus of Canadian parliamentary proceedings (Linguistic Data Consortium, 1997), containing a total of slightly more than one million words, approximately half in cach language. Word-level alignment between French and English was performed using a dictionary containing entries derived statistically from the full Hansard corpus, augmented by the ARTTI, French-English dictionary (ARTFL Project, 1998). 'This dictionary was used for all EBMT and clustering runs.

The effects of varying the amount of training texts were determined by further splitting the training corpus into smaller segments and using differing numbers of segments. For each

\begin{tabular}{|c|c|}
\hline Clust & Mombers \\
\hline 238 & $\begin{array}{l}\text { HISIOIRE HISTORY } \\
\text { ECONOMIE ECONOMY }\end{array}$ \\
\hline 260 & $\begin{array}{l}\text { CERTAINEMENT CLRTAINLY } \\
\text { CERTAINEMENT SURELY } \\
\text { CERTES SURELY } \\
\text { JAMAIS NEVER } \\
\text { PAS NOT } \\
\text { PEUT-ETRE MAY } \\
\text { PROBABLIEMENT PROBABLY } \\
\text { QUE ONLY } \\
\text { RIIEN NOTHING } \\
\text { SUREMENT CERTAINLY } \\
\text { SUREMENT SURELY } \\
\text { VRAIMENT REALLY }\end{array}$ \\
\hline 348 & $\begin{array}{l}\text { CONSERVATEUR CONSERVATIVE } \\
\text { CONSERVATEUR TORY } \\
\text { DEMOCRA'TTQUE DEMOCRATIC } \\
\text { DÉMOCRATTQUL NDPP } \\
\text { LIBÉRAL LIBERAL }\end{array}$ \\
\hline 522 & $\begin{array}{l}\text { DERNIERRS LAST } \\
\text { DERNIERTS PAST } \\
\text { DERNIERES RECENT } \\
\text { PROCHAINIS NEXT } \\
\text { QUELQUES FEW } \\
\text { QUELQUES SOME }\end{array}$ \\
\hline 535 & $\begin{array}{l}\text { AVONS HAVE } \\
\text { SOMMES ARE }\end{array}$ \\
\hline 1375 & $\begin{array}{l}\text { ELECTORALE CAMPAIGN } \\
\text { ELECTOR } \triangle \mathrm{L} \text { FIECTION }\end{array}$ \\
\hline 1386 & $\begin{array}{l}\text { FÉDÉRALES-PROVINCIALLS } \\
\text { FEDERAL-PROVINCIAI } \\
\text { INDUSTRIELIES INDUSTRIAI, } \\
\text { OUVRISRES LABOUR }\end{array}$ \\
\hline 1528 & $\begin{array}{l}\text { FACON EVENT } \\
\text { EVIDENCE CLEARLY } \\
\text { EVIDENCE OBVIOUSIY }\end{array}$ \\
\hline 1563 & $\begin{array}{l}\text { HOMMISS POLITICIANS } \\
\text { PRISONNITRS PRISONERS }\end{array}$ \\
\hline 1652 & $\begin{array}{l}\text { RETOUR BACK } \\
\text { REYINIR BACK }\end{array}$ \\
\hline 2008 & $\begin{array}{l}\text { CONVENU AGREED } \\
\text { SIGNE SIGNED } \\
\text { VU SEEN }\end{array}$ \\
\hline 21.82 & 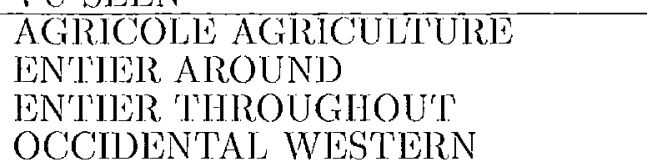 \\
\hline 2472 & $\begin{array}{l}\text { AVIEUGLES BLIND } \\
\text { CHAUSSURES SHOES } \\
\text { CONSTRUC'REURS BUULIDERS } \\
\text { PENSIONNES PENSIONERS } \\
\text { RETRAITIES PENSIONERS } \\
\text { VETTEMTENTS CLOTHING }\end{array}$ \\
\hline 3539 & $\begin{array}{l}\text { POISSON FISII } \\
\text { PORC PORK }\end{array}$ \\
\hline
\end{tabular}

Figure 2: Sample Clusters 
run using clustering, the first $K$ segments of the corpus are concatenated into a single lile, which is used as input for both the clustering program and the EBMT' system. 'The clustering program is run to determine a set of equivalence classes, and these classes are then provided to the ISMMl' system along with the training examples to be indexed. Ileld-out Ilansard text (approximately 15,000 words) is then translated, and the percentage of the words in the test text for which the IBMM' system could find matches and generate a translation is determined.

To test the eflects of adding seed clusters, a set of initial clusters was generated with

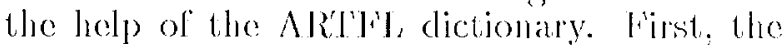
500 most froquent words in the million-word Hansard subset (excluding punctuation) wore extracted. 'These ter'ms were then matched against the ARTTI, dictionary, removing those words which had multi-word tumslations as well as several which listed multiple parts of spech for the same transtation (multiple parts of specch can only be used if the corresponding translations are distinct from each other). 'The remaining 120 translation pairs, tagged for part of speech, were then converted into secel clusters and provided to the clustering program. 'To facilitate experiments using the ple-existing production-1ule grammar, five additional translation pairs from the manually-generated equivalence classes were added to provide seeds for five equivalence classes which are not present in the dictionary.

\section{$5 \quad$ Results}

The method described in this paper does (subjectively) a very good job of clustering like words together, and using the clusters to generalize IBMM' gives a considerable boost to the performance of the eibMT system.

ligure 2 shows a sampling of the smalles clusters generated from 1.1 million words of Hansard text. While the members of a cluster are often semantically linked (as in cluster 348 , which contains types of political partics, or cluster 3539), they need not be. 'T'hose clusters whose members are not semantically linked gencrally contain words which are all the same part, of speech, number, and gender (as in cluster 2472, which contains exclusively plural nouns) - but as will be discussed in the next section, even those clusters whose nembers are totally unrelated may be useful and correct. One fairly common occurrence among the smaller clusters is that various synonymous transtations of a word (from either source or target language) will cluster together, as in cluster 1652. This is particularly useful when the target-language word is the same, as this allows various ways of expressing the sane thing to be translated when any of those forms are present in the training corplus.

Higure 3 shows how adding antomaticallygenerated equivalence classes substantially increases the coverage of the EBMT system. Altermativoly, much less toxt is required to reach a specific level of coverage. The lowest curve in 1.he graph is the perecentage of the 15,000 -word test text for which the IBMM system was able to generate translations when using strict lexial matching against the training corpus. 'The top-most curve shows the best performance previously achieved using both a large set of equivalonce classes (in the form of tagged entries from the ARTl' dictionary) and a production-rule grammar (Jrown, 1999). Of the two conter curves, the lower is the performance when gencralizing the training corpus using the equivalence classes which were automatically generated from that same text, and the upper shows tho performance using clustering with the 125 soed pairs.

As can be seon in ligure $3,80 \%$ coverage of the test text is achieved with less than 300,000 words using manually-created generalization information and with approximately 300,000 words when using alutomaticallycreated generalization information, but requires 1.2 million words whon not using generalizatjon. $90 \%$ coverage is reached with less than 500,000 words using mantally-created information and should be reached with less than 1.2 million words using automatically-created gencralization inlormation, versus 7 million woreds withont generalization. 'This reduction by a factor of four to live in the amount of text is accomplished with litite or no degradation in the equality of tho tramslations. Adding a small amount, of knowledge in the form of 425 seced pairs reduces the recuired traning text even further; this can largely be attributed to the merging of clusters which would otherwise have remained distinct, thus increasing the level of generalization.

Adding the production-rule grammar to the soeded clustering had little effect. When using more than 50,000 words of training text, the increase in coverage from adding the grammar was negligible, and even with the smallest training corpora the increase was very modest.

Using the same thresholds that were used in the fully-automatic case, clustering on 1.1 million words cxpands the initial 425 word pairs in 37 clusters to 3209 word pairs, and adds an additional 555 word pairs in 140 further nontrivial clusters. 'This compares very favorably 


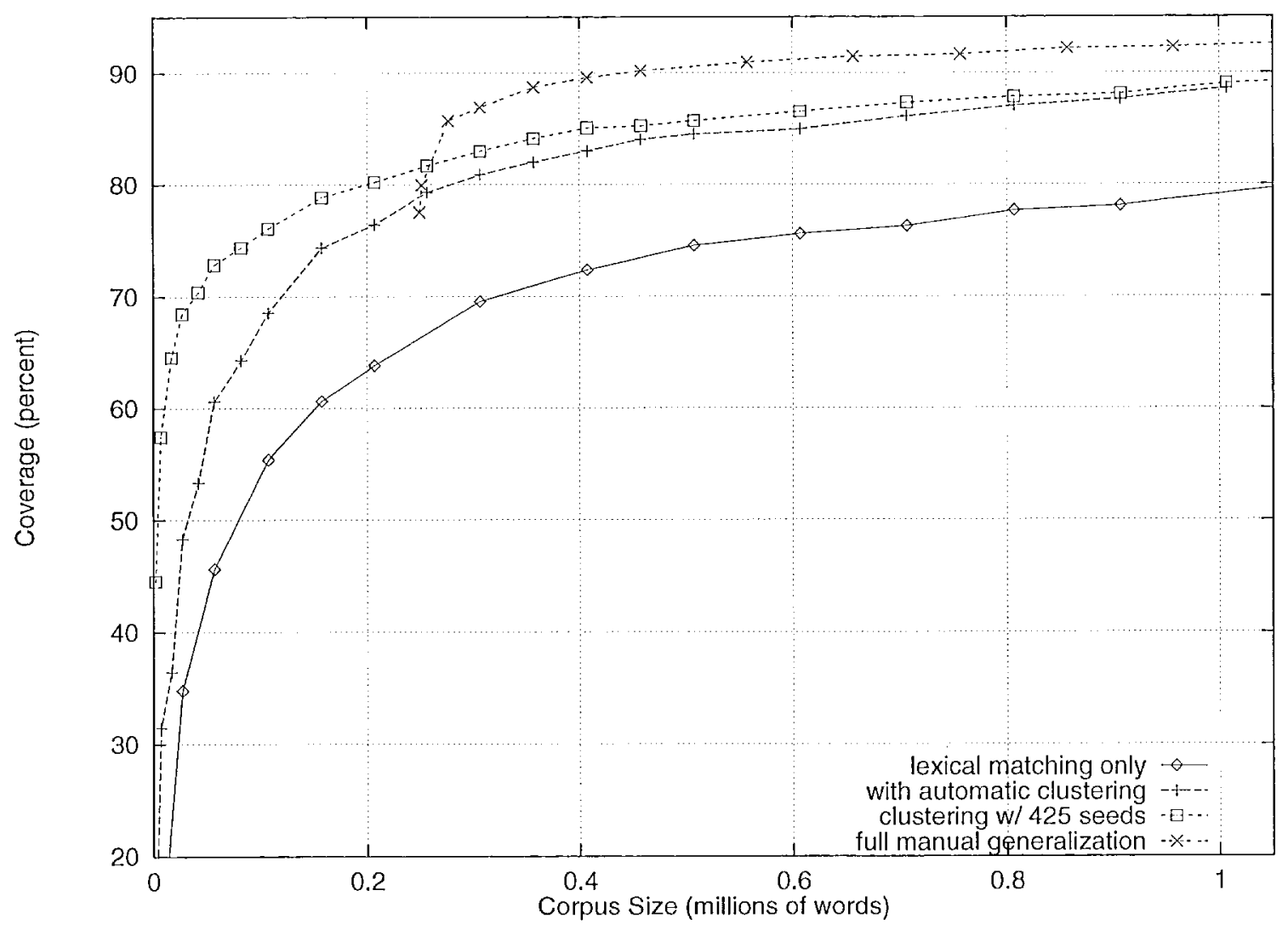

Figure 3: EBMT' Performance with and without Generalization

to the 3506 word pairs in 221 clusters found without sceding.

The program also runs reasonably quickly. The step of creating context term vectors converts approximately 500,000 words of raw text per minute on a $300 \mathrm{MHz}$ processor. For agglomerative clustering, the processing time is roughly quadratic in the number of word pairs, with a theoretical cubic worst case; the 17,527 distinct word pairs found from the million-word training corpus require about 25 minutes to cluster.

\section{Discussion}

One statement made carlier deserves clarification: the members of a cluster need not be related to each other in any way, either syntactically or semantically, for a cluster to be useful and correct. This is because (absent a grammar) we do not care about the features of the words in the cluster, only whether their translations follow the same pattern.

$\Lambda \mathrm{n}$ illustration based on actual experience is useful here. In early testing of the groupaverage clustering algorithm with sceding, tho <conjunction> seed class of "and" and "or" was used. Clustering augmented this seed class with "" (comma), "in", and "by". One call casily see that the comma is a valid member of the class, since it takes the place of "and" in lists of items. But what about "in" and "by", which are prepositions rather than conjunctions? If one considers the translation pattern

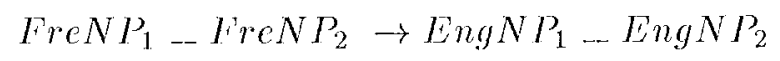

it becomes clear that all of the terms in the expanded class give a correct translation when placed in the blank in this pattern. Indeed, one could imagine a production-rule grammar geared toward taking advantage of such common translation patterns regardless of conventional linguistic features.

\section{Conclusion and Future Work}

Using word clustering to automatically generalize the example corpus of an EBMT system can provide the majority of the improvement which can be achieved using both a manuallygenerated set of equivalence classes and a production rule grammar. The use of a set of small initial equivalence classes produces a substantial further reduction in training text at a very low cost (a few hours) in labor. 
An obvious extension to using sood clusters is to 11 se the result of a clustering run as the initial soed for a second iteration of clustering, since the additional generalization of local contexts enabled by the larger seed clusters will permit additional expansion of the clusters. For such iterative clustering, all but the last round should presumably use stricter thresholdis, to avoid adding too many irrelevant menbers to the clusters. Preliminary experiments have been inconclusive ... although the result of a second iteration contains more terms in the clusters, liBM'T performance does not seom to improve.

More sophisticated clustering algorithens such as k-means and doterministic annoaling may provide better-cuality clusters for better performance, at the expense of increased processing time.

'This approach to gencrating equivalence classes should work just as woll for phrases as for single words, simply by modilying the conversion step to create context, vectors for phrases. This enhancement wondd eliminate the curlent. limitation that translation paiss to be clustered must be single words in both languages. Work on this modification is currently moder way.

An interesting future experiment, would be foregoing grammas rulas based on standard grammatical features such as part of spoech, and instead creating a grammar guided by the clusters found fully automatically (without, secding) from the example text. The recent work by Mc'lait and 'lrujillo (1999) on oxtracting translation patiterns would appear to be a perfect complement, as they are in effoct finding context strings with open slots, while the work described here finds the filless lou those slots. Given the ability to learn such a grammat withont manual intervention, it would become possible to create an liBMT' system using generalized examples from nothing more than parallel text, which for many language pairs could also be acquired almost fully automatically by crawling the World Wide Web (Resnik, 1998).

\section{References}

ARTPL Project. 1998. French-English bictionary. Project for American and Trench Research on the 'Treasury of the lirench language, University of Chicago. http://humanities .uchicago edu/ARTFL .html.

Sergio Barrachina and Juan Miguel Vilar. 1999. Bilingual Clustering Using Monolingual Algorithms. In Procedings of the Jighth International Conference on Theoretical and Melhodological Issues in Machine Translation (TMI99), pages 77-87, Chester, England, August.
Peter Brown, J. (Cocke, S. Della Piotra, V. I)ella Picta, l'. Jelinck, J. Iaflerty, R. Mercer, and P. Roossin. 1990. A Statistical Approach to Machine 'Tanslation. Compulational Linguislics, $16: 7985$.

Rall 1). J3rown. 1997. Automated Dictionary lixtraction for" "Knowledge-Iree" JixampleBased Translation. In Procedings of the Sconth Intenalional Conference on Theorelical and Methodolorical Issues in Machine Translation (1MJ-97), pages 111 118, Santa lie, New Mexico, July. http://wWw . cs. cmu .edu/"ralf/papers .html.

Rall I). Brown. 1999. Adding Iinguistic Knowledge to a Irexical Ixample-l3ased 'Translation System. In Proccedings of the Lighth Inlemiational Conference on Theoretical and Melhodological Issues in Machine Thanslation (TMI-99), pages 22 32, Chester, Fingland, August. http://ww. cs. cmu. edu/"ralf/papers.html.

Jinguistic 1)ata Consortium. 1997. Ilansand Conpus of Parallel linglish and fircnch. linguistic 1)ata Consortium, December. http : / /www. ldc . upenn . edu/.

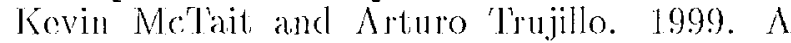
language-Neutral Sparso-I)ata Algorithm for Wxtracting 'Translation Patierns. In Procedings of the Eighth International Conference on Theorctical and Mrthodological lssucs in Marhine Jranslation (TM-99), pages 98 108, Chester, England, August.

Philip Resnik. 1998. Parallel Strands: A l'reliminary Investigation into Mining the Wob for Bilingual 'lext. In David Harwell, Iaurie Gorber, and Lduard Hovy, editors, Machine Translation and the Information Soup: Third Conference of the Association for Mrechine Translation in the Americas (AMTA-98), volume 1529 of Lecture Notes in Arlificial Intelligence, pages 72 82, Janghorne, Pennsylvania, Octolser. Springer.

'Tony Veale and Andy Way. 1997. Gaijin: $\Lambda$ 'Complate-I)riven Bootstrapping Approach to Example-Based Machine Translation. In Proccedings of the NeMNIP'g\%, New Methods in Natural Langauge Processessing, Solia, Bulgaria, September. http://www. compapp.dcu.ie/ tonyv/papers/gaijin.html.

Ellen M. Voorhees. 1986. Implementing $\Lambda g-$ glomerative Hicrarchical Clustering Algofithms for Use in Document Retrieval. Informalion Processing and Management, $22(6): 465-476$. 